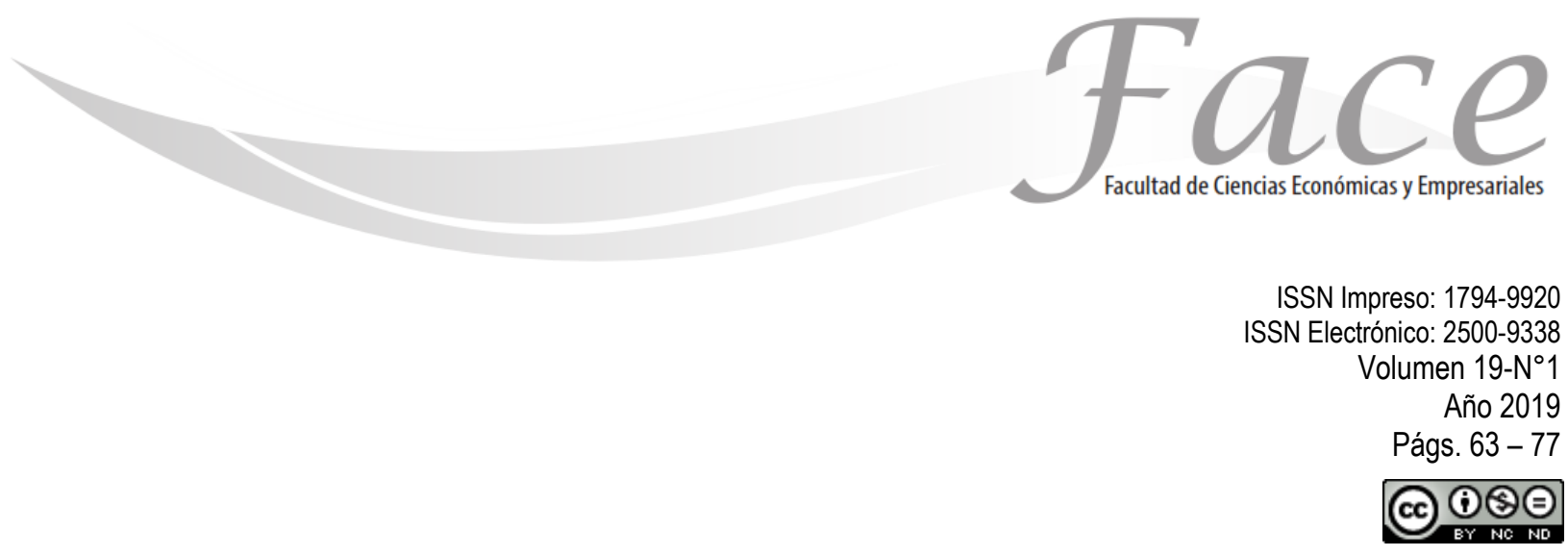

\title{
RESPONSABILIDAD SOCIAL EN INSTITUCIONES DE EDUCACIÓN: PROPUESTA DE MEDICIÓN
}

\author{
José Felipe Ojeda Hidalgo * \\ ORCID: https://orcid.org/0000-0001-8714-1292
}

Fecha de Recepción: Junio 29 de 2019

Fecha de Aprobación: Octubre 4 de 2019

\section{Resumen:}

La presente investigación desarrollo un instrumento para medir la responsabilidad social en instituciones de educación superior, integrando diversas teorías que han emergido en el transcurso del siglo XXI, fue posible identificar diez variables a través de un instrumento de 42 ítems que demostró consistencia interna y validez del constructo. El estudio muestra las correlaciones, regresiones y algunos comparativos con las variables sociodemográficas cuyos resultados se discuten en el cuerpo del trabajo.

Palabras clave: Responsabilidad social, Instituciones de educación superior, Medición

\footnotetext{
* Dr. en Administracion, profesor - investigador de tiempo completo en la Universidad Politécnica de Guanajuato, docente y miembro del comité doctoral de la Universidad del Centro del Bajio y de la Universidad Cuahutemoc - Aguascalientes, profesor invitado en la Universidad De La Salle - Bajio - Salamanca, Universidad de Occidente - Los Mochis y Universidad de Celaya. Contacto: jojeda@upgto.edu.mx
} 


\title{
SOCIAL RESPONSIBILITY IN EDUCATION INSTITUTIONS: MEDICINE PROPUTER
}

\begin{abstract}
:
This research developed an instrument to measure social responsibility in higher education institutions, integrating various theories that have emerged in the course of the XXI century, it was possible to identify ten variables through a 42-item instrument that demonstrated internal consistency and validity of the construct. The study shows the correlations, regressions and some comparisons with the sociodemographic variables whose results are discussed in the body of work
\end{abstract}

Keywords: Social responsibility, Institutions fo higher education, Measurament

\section{RESPONSABILIDADE SOCIAL NAS INSTITUIÇÕES DA EDUCAÇÃO: PROPUTADOR DE MEDICINA}

\section{Resumo:}

Esta pesquisa desenvolveu um instrumento para medir a responsabilidade social nas instituições de ensino superior, integrando várias teorias que surgiram ao longo do século XXI. Foi possível identificar dez variáveis através de um instrumento de 42 itens que demonstrou consistência interna e validade do construto. . O estudo mostra as correlações, regressões e algumas comparações com as variáveis sociodemográficas cujos resultados são discutidos no corpo do trabalho.

Palavras-chave: Responsabilidade Social, Instituições de Ensino Superior, Medição. 


\section{INTRODUCCIÓN:}

La responsabilidad social es un tema que ha existido, en sus primeras fases, desde el principio de las civilizaciones, es posible rastrear escritos que hacen referencia a esas preocupaciones, como Los Gathas, de Zaratustra, o el código de Hammurabi, o la Ética a Nicómaco (Ojeda, 2015a). Las fases son posibles distinguirlas en función de la ética, el equilibrio social, el bien común, el altruismo y la filantropía (Ojeda, Vega, Vera, 2015).

El concepto de responsabilidad social como idea ha sufrido cambios dependiendo del ángulo histórico y epistémico desde el cual se esté revisando, ello como elemento organizacional general, como idea básica, aterrizada en las instituciones de educación, se puede rastrear al Congreso Internacional de Estudiantes Americanos de 1908 en el cual se discutió sobre la función social de la universidad y que sirvió de base para que 10 años después, durante la llamada Reforma de Córdova de 1918 se estableciera la llamada extensión universitaria, concepto que fue retomado durante la Primer Conferencia Latinoamericana de Extensión Universitaria y Difusión Cultural de 1950. En ese trayecto se ha cuestionado si la razón social de las universidades es solo la extensión, es decir, ir más allá de la investigación y la docencia, como se estableció durante el Primer Congreso de Universidades Latinoamericanas en 1949 o si por el contrario, la extensión es únicamente una de las funciones que corresponden a un cuerpo social más complejo de estas instituciones sociales llamadas universidad (Tünnermann, 2003).

A finales del siglo $X X$ y principios del XXI existieron iniciativas para que las universidades formaran diplomados altamente cualificados para que participen activa y responsablemente en la sociedad se buscó su integración activa a través de la resolución 57/254 en el llamado decenio de la educación para el desarrollo sostenible y en la ratificación de la los objetivos y misiones del 2009, al ser llamadas, las universidades, a contribuir desde la educación al desarrollo sustentable.

En este devenir histórico vale la pena resaltar la iniciativa Universidad Construye País, que pone sobre la mesa de discusión el concepto de responsabilidad social, como un elemento adicional y no dado por generación espontánea dentro de las instituciones de educación superior.

Otra iniciativa que es pertinente mencionar es la de la Asociación de Universidades Confiadas a la Compañía de Jesús en América Latina (AUSJAL), que tienen como misión "potenciar la habilidad y efectividad de las universidades de la Red AUSJAL para responder, desde el ejercicio de sus funciones sustantivas, a las necesidades de transformación de la sociedad en clave de justicia, solidaridad y equidad social" (Gargantini, 2019:1) y que al mismo tiempo propone líneas específicas de trabajo para abordar el tema de la responsabilidad social dentro de las instituciones de educación superior: a) Definir el enfoque de la responsabilidad social y las líneas políticas a implementar entre las universidades adherentes, b). Desarrollar una metodología de auto diagnóstico, seguimiento y evaluación que permita conocer el estado de la responsabilidad social en las universidades de AUSJAL, c). Identificar, determinar, planificar y presupuestar propuestas de fortalecimiento instituciones de la gestión de la responsabilidad social en las universidades participantes, y d). Realizar el seguimiento y evaluación de las acciones de apoyo recomendadas para el fortalecimiento de la gestión de la responsabilidad social en cada una de las universidades participantes de la Red.

El concepto de responsabilidad ha vuelto a cobrar importancia debido a los grandes escándalos corporativos y empresariales que se comenzaron a suceder a comienzos del siglo XXI (Montaño, 2012) o los sucedidos en fechas más recientes y de los cuales las redes sociales han dado cuenta de manera muy puntual (Ojeda, 2015b)1. Aun con estas evidencias, hubo un tiempo en el que se pensó que las instituciones de educación estaban fuera de la mano de los escándalos y de acciones contrarias a la responsabilidad social, hasta que al menos, en México, sucedió la llamada estafa maestra (Ojeda, 2018a) ${ }^{2}$ hecho que puso en entredicho la "pureza" de las instituciones educativas e hizo que varias personas se cuestionaran las llamadas estrategias de responsabilidad social que se promueven en las instituciones de educación superior.

\section{REVISIÓN DE LA LITERATURA:}

Como ya se ha establecido el devenir histórico de la responsabilidad social va casi a la par del devenir histórico de la humanidad, desde el Código de Ur - Nammu (2100 a.C), pasando por el Código de Hammurabi (1750 a.C.), Los Gathas de Zaratustra (1776 a.C.), las sociedades se han preocupado por elementos que ahora llamamos acciones de responsabilidad social.

En este proceso evolutivo, la responsabilidad social ha evolucionado por diversas dimensiones que han colaborado de una forma u otra, en ir configurando este concepto (Ojeda, Vega y Vera, 2017), y es precisamente por este proceso que en la actualidad la responsabilidad social tiene acercamientos diversos, desde enfoques igualmente variados, de ahí que para De la Cruz y Saisa (2008) los enfoques sean consecuencialista, contractual y

\footnotetext{
${ }^{1}$ El autor hace referencia a los casos de Pride, Noble Group, ABB, Siemens, Eni, KBR, GE, Goldman Sachs, Walmart, First Majestic, OHL, etc.

${ }^{2}$ El autor hace referencia al desvió de recursos públicos en el cual se vio involucradas la menos la Universidad Autónoma del Estado de México, Universidad Autónoma del Carmen, Universidad Autónoma del Estado de Morelos, Universidad Popular de la Chontalpa, Universidad Autónoma Juárez de Tabasco, Universidad Politécnica del Golfo de México, Instituto Técnico Superior de Comalcalco y la Universidad Tecnológica de Tabasco.
} 
prospectivo, para Rodríguez (2010) los enfoques son el académico tradicional, el corporativo, el empresarial e instrumental y el global, pluralista y responsable, para Gaete (2011) dichos enfoques son el gerencial, el transformacional y el normativo, para Larran y Andrades (2015) son el de la teoría de la agencia, los stakeholder, la legitimidad, el institucional y el de recursos y capacidades; con esta multitud de enfoques no es de sorprenderse que existan, igualmente, gran variedad de conceptos sobre el fenómeno de responsabilidad social dentro de las instituciones de educación superior y universidades (Arocena y Sutz, 2001; Urzúa, 2001; Jimenéz, 2002; Guédez, 2003; Vallaeys, 2005; Centeno, 2006; Cavero, 2006; Sánchez, Herrera, Zarate y Moreno 2007; Delgado, 2007; Martínez, Mavarez, Rojas, Rodríguez y Carvallo, 2008; Cevallos, 2008; Martínez y Picco, 2008; Vallaeys, De la Cruz y Saisa, 2009; Hernández y Saldarriaga, 2009; Boelen, 2009; Rendueles, 2010; Díaz y Facal, 2011; Gaete, 2011; García y Alvarado, 2012) si bien los conceptos tienen elementos en común, de la misma forma presentan elementos que hace que parezca que cada autor está hablando de una responsabilidad social distinta en cada institución de educación superior o universidad.

Con estos antecedentes no es de sorprender que las acciones de responsabilidad social se comprendan de formas distintas en distintas instituciones, y que emerjan varias dimensiones que complican el estudio $y$ comparación del fenómeno (Ojeda, 2013a; Ojeda, 2013b; Ojeda, 2014; Ojeda, 2015; Ojeda y Álvarez, 2015; Arredondo y Ojeda, 2015; Ojeda, Silva y Álvarez, 2015; Ojeda, Vega y Vera, 2017; Ojeda, Álvarez y Arredondo, 2018).

Aldeanueva y Arrabal (2018) encontraron que las acciones de responsabilidad social en las instituciones de educación superior se enfocan en el impacto social y el compromiso social, a través de acciones de voluntariado y solidaridad, fomentando la ética, el compromiso, la moral y la equidad, se enfocan sobre todo en los temas medioambientales y se ajustan a los indicadores de cooperación internacional o de desarrollo establecidos por el gobierno en turno, manejan dentro de su discurso la frases de responsabilidad social, aunque no lo entiendan del todo a que se refieren.

Algunos ex rectores, han manifestado sus visiones y preocupaciones de hacia dónde debiera ir el futuro de la responsabilidad social dentro de las instituciones de educación superior, de ahí que por ejemplo Juan Carlos Romero Hicks (ex rector de la Universidad de Guanajuato) enfatiza la ausencia de leyes, las políticas públicas inadecuadas, la necesidad de realizar rediseños institucionales y un sano manejo de los recursos económicos, por otro lado Ernesto Fernández Fassnacht, ex secretario general de la Asociación Nacional de Universidades e Instituciones de Educación Superior, resalta que las Universidades les hace falta promover el pensamiento crítico, generar una ciudadanía activa, establecer de forma efectiva la formación en competencias, fortalecer la confianza de las partes interesadas de la universidad, que los beneficios generadas por dichas universidades, estén bien distribuidas geográficamente, promover la creación de mayores espacios de inclusión, vigilar que el gobierno diseñe los incentivos adecuados para que la responsabilidad social ocurra y que la cobertura de sus beneficios crezcan.

Por su parte, Enrique Rivera Villa, ex Director del Instituto Politécnico Nacional enfatiza la necesidad de la trasparencia y la mejora continua, la rendición de cuentas, el liderazgo claro, la congruencia institucional y el buen equipo de trabajo. Felipe Cuamea Velazquez, ex Rector de la Universidad Autónoma de Baja California, es más concreto y solo se enfoca en transformar las instituciones y la necesidad de transparencia, finalmente, Julio Rubio Oca, ex Rector de la Universidad Autónoma Metropolitana, describe tres líneas a seguir, la transparencia, la rendición de cuentas y la imagen responsable (Ocegueda, Mungaray, Rubio y Moctezuma, 2016) 3

Con estas visones, que si bien son importantes, dada la categoría y experiencia de los personajes que las emiten, académicamente hablando, carecen de rigor académico y de concepto, si bien la transparencia es un elemento fundamental y las dimensiones medioambientales se han atendido de una u otra forma, quedan en el aire los temas referentes a las prácticas laborales, la tercerización del trabajo sigue siendo un tema pendiente, los productos chatarras vendidos dentro de estas instituciones (papas fritas, golosinas disfrazadas de pan dulce, gaseosas, etc.) son temas que no se discuten, de ahí que algunas instituciones evidencian prácticas que son importantes para el avance en cuanto a la responsabilidad social, terminan siendo acciones aisladas, carentes de integralidad en cuanto al verdadero espíritu de la responsabilidad social.

Pérez y Vallaeys (2016) recogieron las mejores prácticas de algunas universidades que sirven de testimonio "[...] que muestran reflexiones, iniciativas y buenas prácticas en torno al ejercicio de la RSU [...]" (p. 13) y que sin embargo evidencian al mismo tiempo la gran brecha que falta por saldar para lograr la verdadera responsabilidad social desde la universidad. Si se comparan dichas acciones con las materias de interés de la responsabilidad social dictadas por la iniciativa ISO 26000, que ha demostrado ser la más adecuada para cualquier organización (Cruz, Rositas y García, 2013; Ojeda y Lira, 2014) es posible observar el largo camino que falta por recorrer.

\footnotetext{
${ }^{3}$ La información ha sido interpretada a partir de los discursos que los ex rectores, durante la reflexión sobre la responsabilidad social de la universidad mexicana, evento organizado por el Centro de Estudios sobre la Universidad de la Universidad Autónoma de Baja California y recogidos en el texto la responsabilidad social de la universidad mexicana a mitad del siglo XXI
} 
Tabla 1.

Comparaciones de las acciones de responsabilidad social de la universidad vs las materias de interés de la ISO26000

\begin{tabular}{|c|c|c|}
\hline Universidad & Acción & $\begin{array}{l}\text { Dimensión de la } \\
\text { responsabilidad social }\end{array}$ \\
\hline \multirow[t]{2}{*}{ Universidad Tecnológica de Torreón } & $\begin{array}{l}\text { Universidad incluyente para personas con discapacidad } \\
\text { motriz, visual, auditiva y de lenguaje }\end{array}$ & Derechos humanos \\
\hline & Campañas con impacto social & $\begin{array}{l}\text { Participación activa y desarrollo } \\
\text { de la comunidad }\end{array}$ \\
\hline \multirow[t]{3}{*}{ Universidad Autónoma de Chihuahua } & $\begin{array}{l}\text { Programa de transversalización de la equidad de género } \\
\text { en la Universidad Autónoma de Chihuahua }\end{array}$ & Derechos humanos \\
\hline & Distintivo Empresa Familiarmente Responsable ${ }^{1}$ & $\begin{array}{l}\text { Derechos humanos } \\
\text { Prácticas laborales }\end{array}$ \\
\hline & $\begin{array}{l}\text { Programa universitario de gestión de residuos sólidos no } \\
\text { peligrosos }\end{array}$ & Medioambiente \\
\hline \multirow{3}{*}{$\begin{array}{l}\text { Instituto Tecnológico Superior de } \\
\text { Oriente del Estado de Hidalgo }\end{array}$} & Sistema de gestión ambiental & Medioambiente \\
\hline & $\begin{array}{l}\text { Aplicación móvil para la atención integral a la equidad de } \\
\text { género }\end{array}$ & Derechos humanos \\
\hline & AGI reforest & Medioambiente \\
\hline \multirow[t]{2}{*}{ Universidad Autónoma de Yucatán } & $\begin{array}{l}\text { Programa institucional prioritario "Gestión del medio } \\
\text { ambiente" }\end{array}$ & Medioambiente \\
\hline & Siete proyectos sociales en comunidades de aprendizaje & $\begin{array}{l}\text { Participación activa y desarrollo } \\
\text { de la comunidad }\end{array}$ \\
\hline \multirow[t]{4}{*}{$\begin{array}{l}\text { Universidad Michoacana de San } \\
\text { Nicolás de Hidalgo }\end{array}$} & $\begin{array}{l}\text { La gestión integral y manejo de los residuos eléctricos y } \\
\text { electrónicos: un aporte a la responsabilidad social y } \\
\text { ambiental en la Universidad Michoacana }\end{array}$ & Medioambiente \\
\hline & $\begin{array}{l}\text { Análisis de la huella ecológica en la Universidad } \\
\text { Michoacana de San Nicolás de Hidalgo }\end{array}$ & Medioambiente \\
\hline & $\begin{array}{l}\text { Ecoturismo rural de naturaleza, aprendizaje - servicio: } \\
\text { una contribución de alumnos para el autodesarrollo del } \\
\text { ejido de Huatzirán, en La Huacana, Michoacán }\end{array}$ & Medioambiente \\
\hline & Diplomados de formación de actores en RSU & Prácticas laborales \\
\hline Universidad Autónoma de Coahuila & Líderes jóvenes ambientalistas "ELIA" & Medioambiente \\
\hline Universidad Tecnológica de Tabasco & Programa institucional de valores y actitudes & Derechos humanos \\
\hline $\begin{array}{l}\text { Universidad Autónoma "Benito Juárez" } \\
\text { de Oaxaca }\end{array}$ & $\begin{array}{l}\text { Brigadas culturales y educativas del Instituto de Ciencias } \\
\text { de la Educación de la Universidad Autónoma "Benito } \\
\text { Juárez" de Oaxaca (ICEUABJO) }\end{array}$ & $\begin{array}{l}\text { Participación activa y desarrollo } \\
\text { de la comunidad }\end{array}$ \\
\hline Universidad Autónoma de Guadalajara & Unidad de pacientes en estudio & $\begin{array}{l}\text { Participación activa y desarrollo } \\
\text { de la comunidad }\end{array}$ \\
\hline $\begin{array}{l}\text { Benemérita Universidad Autónoma de } \\
\text { Puebla }\end{array}$ & $\begin{array}{l}\text { Tifloteca biblioteca inclusiva en la Benemérita } \\
\text { Universidad Autónoma de Puebla }\end{array}$ & $\begin{array}{l}\text { Participación activa y desarrollo } \\
\text { de la comunidad }\end{array}$ \\
\hline $\begin{array}{l}\text { Universidad Tecnológica de } \\
\text { Tamaulipas }\end{array}$ & Ayudando aprendo & $\begin{array}{l}\text { Participación activa y desarrollo } \\
\text { de la comunidad }\end{array}$ \\
\hline \multirow[t]{2}{*}{ Universidad Autónoma de Chiapas } & $\begin{array}{l}\text { Los Bravos: Integración de estudiantes en proyectos de } \\
\text { investigación y transferencias de tecnologías }\end{array}$ & $\begin{array}{l}\text { Participación activa y desarrollo } \\
\text { de la comunidad }\end{array}$ \\
\hline & Unidades de vinculación docente & $\begin{array}{l}\text { Participación activa y desarrollo } \\
\text { de la comunidad }\end{array}$ \\
\hline
\end{tabular}

Fuente: Elaboración propia con información de Pérez y Vallaeys (2016) 
Queda claro que las instituciones de educación superior han enfocado sus esfuerzos en las dimensiones de responsabilidad social que permiten cierto lucimiento externo, sin embargo, las dimensiones que se evalúan hacia el interior están, hasta cierto punto olvidadas, en las prácticas evidenciadas no se denotan acciones de gobernabilidad, prácticas justas de operación y asunto de los consumidores; si bien es cierto que algunas de estas instituciones, efectivamente tienen prácticas en estos rubros que se acaban de calificar de "olvidados", dichas prácticas obedecen a protocolos establecidos en legislaciones 0 en procedimientos adherentes a alguna declaratoria y que por tanto son de carácter obligatorio, y es, precisamente esta obligatoriedad, la que le quita la característica de responsabilidad social a dichas acciones, ya que la responsabilidad social es voluntaria, es decir, comienza donde la legalidad se satisface.

\section{Medición de la responsabilidad social en instituciones de educación superior}

Al inicio del siglo XXI, el estudio de la responsabilidad social en instituciones de educación superior era aún limitado y de carácter descriptivo, se había centrado principalmente en las percepciones de los estudiantes, docentes 0 autoridades (Montgomery y Ramus, 2004) 0 en algunos aspectos curriculares (Gordon y Geraldi, 2005; Matten y Moon, 2004). Existía muy poco sobre los sistemas de gestión, aplicación y evaluación de los modelos de responsabilidad social, o un sistema conceptual definitivo 0 metodologías de evaluación y medición (Vallaeys, 2008).

Los estudios se enfocaban en evaluar las percepciones de los estudiantes y las causas del concepto ( Matten y Moon, 2004; Ibrahim, Agneldis y Howard, 2006; Domínguez y López, 2009), otros estudios centraban sus discusiones en analizar la oferta de cursos de responsabilidad social empresarial en la currículo de las universidades (Seto Paimes, Domingo - Vernis y Rabasa - Figueroa, 2011; Castillo, 2010; Holdsworth, Wyborn, Bakessy y Thomas, 2008; Buchan, Spellerberg y Blum, 2008; Ciura y Filho, 2006), o se presentaban estudios de casos basados en universidades concretas (Ferrer - Balas, Adachi, Banas, Davidson, HoshiKoshi, Mishra, Motoda, Onga y Ostwald, 2008; Hammond y Churchman, 2008; Atakan y Eker, 2007)

Existen otros estudios que enfatizan el compromiso de la universidad con comunidades socialmente excluidas 0 indígenas, en las que se plantea el rol de la universidad como conector entre la educación, derechos cívicos, mercado laboral, entre otros, y dichas comunidades (Benneworth, 2013). Campbell y Christie (2008) resaltan las acciones de comunicación, colaboración, incorporación de conocimientos y compromiso a largo plazo con estas comunidades.
El hecho de que las universidades se asuman como socialmente responsables, por el hecho de enseñar responsabilidad social en lugar de analizar y revisar el nivel de desempeño y conductas éticas, han logrado que algunas sean vistas como instituciones incongruentes, puesto que enseñan el deber ser, pero ellas no lo hacen (Hill, 2004).

La preocupación sobre la responsabilidad social en las instituciones de educación superior es real y significativa y se ha detonado todo un movimiento para entender y delimitar esta responsabilidad, cómo se debe actuar y hasta qué punto se es responsable de sus procesos (Sanje y Senol, 2012).

Parte de la discusión es el cómo debe medirse, la igual que la responsabilidad social en las organizaciones, la responsabilidad social en las instituciones de educación superior tiene una diversidad conceptual, como ya se ha discutido líneas arriba, para Vallaeys (2008) las instituciones de educación superior tienen un vínculo inexorable con la sociedad, a la vez que todos los actos de las instituciones de educación superior tienen un impacto sobre la sociedad.

Para algunos autores la responsabilidad social en las instituciones de educación superior y la responsabilidad social organizacional deben ser radicalmente distintas, ya que la responsabilidad social que práctica una empresa, incluyendo la sector educativo, es una estrategia que busca aumentar la rentabilidad, a través de un marketing, que genere una mejor imagen pública y social, aunque sean incapaces de resolver los problemas de exclusión social (Dias, 2008; Dupa, 2005; Vallaeys, De la Cruz y Saisa, 2009), sin embargo ante estas afirmaciones ya se ha establecido que:

[...] la responsabilidad social debe hacerse al margen del impacto propagandístico de la acción [...] corrientemente se proclama que la responsabilidad social es un buen negocio y que la ética es rentable. Cuando se hace uso de estas expresiones, ambos aspectos se convierten en intereses y beneficios económicos que terminan por desvalorizarse. Vender ética diciendo que es rentable, 0 vender la responsabilidad social afirmando que es un buen negocio, equivale a asumir una visión antiética de la ética, y una interpretación irresponsable de la responsabilidad social. Esas afirmaciones son demagógicas, porque además de manipuladoras y retóricas, no son totalmente ciertas y en consecuencia terminan siendo aseveraciones engañosas (Guédez, 2006: 11 y 75). 
Vallaeys (2008) compara la responsabilidad social de las empresas con la responsabilidad social de las instituciones educativas en cuanto a la necesidad de ambas de gestionar sus impactos, aunque también precisa que las instituciones de educación superior deben ser sensibles a los problemas sociales, así como a los compromisos y acciones que la universidad debe asumir para responder a las exigencias sociales.

No obstante, continúan existiendo posturas que sostienen que las instituciones de educación superior deben tener un discurso propio sobre su responsabilidad, que vaya más allá de su misión, y que sirva como fundamento para comprender cuál sería su responsabilidad social como institución de educación superior (Vallaeys, De la Cruz y Saisa, 2009).

Diversos autores han propuesto una serie de variables para medir la responsabilidad social (Jímenez, 2002; Jímenez, De Ferari, Delpiano y Andrade, 2004; Davidovich, Espina, Navarro y Salazar, 2005; Vallaeys y Carrizo, 2006; Sánchez González, Herrera Márquez, Zárate Moreno y Moreno Méndez, 2007; Vallaeys, 2007; Castañeda, Ruíz, Viloria, Castañeda y Quevedo, 2007; Gómez, De Pelekais y Tirado, 2009 y Ernult, 2011), algunos hacen énfasis en que se trata de indicadores exclusivos de responsabilidad social en instituciones de educación y proponen variables como docencia, extensión e investigación, algunos otros son más holísticos y proponen que, todas las instituciones de educación, toda vez que son organizaciones, tienen las mismas variables que cualquier otro sujeto organizacional, por lo que las variables que las explican son las mismas.

De forma general, las variables que más se han utilizado en la valoración de la responsabilidad social en instituciones de educación son:
Tabla 2.

Variables para medir la responsabilidad social en instituciones de educación superior

\begin{tabular}{|c|c|}
\hline \multirow{3}{*}{$\begin{array}{l}\text { Institución } \\
\text { ANFECA (2016) }\end{array}$} & \multirow{2}{*}{ Variables } \\
\hline & \\
\hline & Docencia \\
\hline & Conocimiento \\
\hline & Organización social y ambiental \\
\hline & Derechos Humanos \\
\hline & Legalidad \\
\hline & Participación ciudadana \\
\hline & Sustentabilidad \\
\hline & Compromiso ético \\
\hline & No corrupción \\
\hline \multirow[t]{12}{*}{ URSULA (2018) } & Buen clima laboral \\
\hline & Campus ecológico \\
\hline & Ética, transparecia, inclusión \\
\hline & Aprendizaje basado en proyectos sociales \\
\hline & Inclusión curricular ODS \\
\hline & Mallas diseñadas con actores externos \\
\hline & Inter y trans disciplinariedad \\
\hline & Investigación en y con la comunidad \\
\hline & $\begin{array}{l}\text { Producción y difusión pública de conocimientos } \\
\text { útiles }\end{array}$ \\
\hline & $\begin{array}{l}\text { Integración de la proyección con formación e } \\
\text { investigación }\end{array}$ \\
\hline & Proyecto co - creados, duraderos, de impacto \\
\hline & $\begin{array}{l}\text { Participación activa en agenda local, nacional e } \\
\text { internacional }\end{array}$ \\
\hline \multirow[t]{9}{*}{ ORSALC (2018) } & Buen gobierno \\
\hline & Clima laboral : Equidad, genero e integración \\
\hline & Medio ambiente y campus verde \\
\hline & Rehumanización \\
\hline & Aprendizaje y servicio \\
\hline & Vinculación y voluntariado \\
\hline & Stakeholders \\
\hline & Educación para el desarrollo sustentable \\
\hline & Ambiente y cultura juvenil \\
\hline \multirow{9}{*}{$\begin{array}{l}\text { Secretaria del Trabajo y } \\
\text { Previsión Social (2016) }\end{array}$} & Ideología central \\
\hline & Condiciones de trabajo y previsión social \\
\hline & Desarrollo de competencias \\
\hline & Comunicación y cooperación \\
\hline & Pertinencia y satisfacción \\
\hline & Dignificación en el empleo \\
\hline & Hostigación laboral \\
\hline & Participación en la comunidad \\
\hline & Vinculación con el medio ambiente \\
\hline \multirow[t]{7}{*}{ CRESE (2016) } & Dirección y comunicación \\
\hline & Justicia salarial y cultura de la legalidad \\
\hline & Calidad de vida en el trabajo y desarrollo personal \\
\hline & Cuidado y desarrollo de la familia \\
\hline & Solidaridad y ayuda a la comunidad \\
\hline & Promoción y cuidado del medio ambiente \\
\hline & Humanización y trascendencia en el trabajo \\
\hline \multirow[t]{5}{*}{ OMERSU (2016) } & Gestión administrativa responsable \\
\hline & Gestión ambientalmente responsable \\
\hline & Producción de conocimiento de la universidad \\
\hline & Formación socialmente responsable de los \\
\hline & $\begin{array}{l}\text { estudiantes } \\
\text { Participación social de }\end{array}$ \\
\hline \multirow[t]{7}{*}{ ISO $26000(2010)$} & Gobernabilidad \\
\hline & Derechos humanos \\
\hline & Medio ambiente \\
\hline & Prácticas laborales \\
\hline & Prácticas justas de operación \\
\hline & Asunto de los consumidores \\
\hline & Participación activa y desarrollo de la comunidad \\
\hline
\end{tabular}

Fuente: Ojeda (2018) 
Si bien es cierto que existe una variedad de variables para medir la responsabilidad social en instituciones de educación superior, también es cierto que existen trabajos que han demostrado que, en este tipo de organizaciones, las variables de la ISO 26000 ya tienen contenidas a las variables propuestas por Jiménez (2002) o Vallaeys (2006) (Cruz, Rositas y Garcia, 2013; Ojeda y Álvarez, 2015 y Ojeda, Vega y Vera, 2017). A partir de ahí es que surge el objetivo de esta investigación que fue desarrollar un instrumento capaz de medir la responsabilidad social en instituciones de educación superior en el estado de Guanajuato.

\section{MÉTODO:}

El planteamiento de esta investigación es cuantitativo, con un diseño no experimental, a través de un alcance correlacional - explicativo, a partir de una muestra de 291 docentes de universidades de nivel superior, públicas y privadas. La población encuestada mostro un $49.5 \%$ de población femenina, $50.5 \%$ masculino, los grados académicos fueron de un $59.3 \%$ de licenciatura, $25.3 \%$ maestría y $15.4 \%$ de nivel doctorado. El $28.6 \%$ procedían de una institución de carácter privado y el $71.4 \%$ de una institución de carácter público, en cuanto a las edades la población mostro una media del 28.26 años con una desviación estándar de 11.03 y una mediana de 25 años.

A través de un análisis factorial exploratorio se probaron 54 ítems con referencia a la responsabilidad social en las instituciones de educación superior, de los cuales

Tabla 3.

Cargas factoriales de los items probados

\begin{tabular}{|c|c|c|c|c|c|c|}
\hline Variable & Item & $\begin{array}{l}\text { Carga factorial } \\
\text { estandarizada }\end{array}$ & $\begin{array}{c}\text { Varianza del } \\
\text { termino de error }\end{array}$ & a Cronbach & $\begin{array}{l}\text { Índice de } \\
\text { Fiabilidad } \\
\text { Compuesta }\end{array}$ & $\begin{array}{l}\text { Indice de } \\
\text { Varianza } \\
\text { Extraída }\end{array}$ \\
\hline \multirow{9}{*}{ Trasparencia } & V36 & 0.840 & 0.294 & & & \\
\hline & V37 & 0.836 & 0.302 & & & \\
\hline & V34 & 0.832 & 0.309 & & & \\
\hline & V35 & 0.821 & 0.326 & & & \\
\hline & V38 & 0.796 & 0.367 & & & \\
\hline & V39 & 0.717 & 0.486 & 0914 & 0.918 & 0586 \\
\hline & V40 & 0.622 & 0.613 & 0.914 & 0.918 & 0.586 \\
\hline & V33 & 0.617 & 0.619 & & & \\
\hline & $\Sigma$ & 6.081 & 3.314 & & & \\
\hline \multirow{8}{*}{ Derechos humanos } & V9 & 0.855 & 0.269 & & & \\
\hline & V8 & 0.855 & 0.269 & & & \\
\hline & V10 & 0.835 & 0.303 & & & \\
\hline & V11 & 0.825 & 0.319 & & & \\
\hline & V12 & 0.792 & 0.373 & 0.914 & 0.928 & 0649 \\
\hline & V14 & 0.742 & 0.449 & & & \\
\hline & V13 & 0.723 & 0.477 & & & \\
\hline & $\Sigma$ & 5.627 & 2.459 & & & \\
\hline \multirow{5}{*}{ Prácticas laborales } & V18 & 0.814 & 0.337 & & & \\
\hline & V17 & 0.759 & 0.425 & & & \\
\hline & V16 & 0.720 & 0.481 & & & \\
\hline & V19 & 0.708 & 0.498 & 0.806 & 0.838 & 0.565 \\
\hline & $\Sigma$ & 3.001 & 1.741 & & & \\
\hline \multirow{5}{*}{$\begin{array}{c}\text { Prácticas justas de } \\
\text { operación }\end{array}$} & V26 & 0.815 & 0.336 & & & \\
\hline & V25 & 0.715 & 0.489 & & & \\
\hline & V24 & 0.693 & 0.520 & & & \\
\hline & V27 & 0.635 & 0.596 & 0.802 & 0.808 & 0.515 \\
\hline & $\Sigma$ & 2.858 & 1.941 & & & \\
\hline \multirow{5}{*}{ Medio ambiente } & V4 & 0.787 & 0.380 & & & \\
\hline & V6 & 0.727 & 0.472 & & & \\
\hline & V5 & 0.663 & 0.561 & & & \\
\hline & V7 & 0.637 & 0.594 & 0.805 & 0.798 & 0.498 \\
\hline & $\Sigma$ & 2.814 & 2.007 & & & \\
\hline \multirow{5}{*}{$\begin{array}{c}\text { Prácticas de recursos } \\
\text { humanos }\end{array}$} & V52 & 0.762 & 0.420 & & & \\
\hline & V53 & 0.754 & 0.432 & & & \\
\hline & V54 & 0.750 & 0.438 & & & \\
\hline & V51 & 0.721 & 0.480 & 0.765 & 0.834 & 0.557 \\
\hline & $\Sigma$ & 2.986 & 1.770 & & & \\
\hline \multirow{4}{*}{$\begin{array}{c}\text { Participación activa y } \\
\text { desarrollo de la } \\
\text { comunidad }\end{array}$} & V46 & 0.838 & 0.297 & 0.770 & 0.822 & 0.608 \\
\hline & V47 & 0.830 & 0.311 & 0.170 & 0.022 & 0.000 \\
\hline & V45 & 0.659 & 0.566 & & & \\
\hline & $\Sigma$ & 2.327 & 1.175 & & & \\
\hline \multirow{4}{*}{ Vinculación } & V22 & 0.761 & 0.421 & & & \\
\hline & V21 & 0.741 & 0.451 & 0773 & 701 & 0557 \\
\hline & V20 & 0.738 & 0.455 & 0.113 & 0.191 & 0.55 \\
\hline & $\Sigma$ & 2.239 & $1 . .328$ & & & \\
\hline \multirow{4}{*}{ Extensión } & V41 & 0.741 & 0.451 & & & \\
\hline & V42 & 0.641 & 0.590 & 0738 & & \\
\hline & V43 & 0.626 & 0.608 & 0.738 & 0.710 & 0.451 \\
\hline & $\Sigma$ & 2.008 & 1.648 & & & \\
\hline \multirow{2}{*}{ Asunto de los usuarios } & V31 & 0.739 & 0.454 & & & \\
\hline & $\frac{\mathrm{V} 32}{\Sigma}$ & $\begin{array}{l}0.614 \\
1.352\end{array}$ & $\begin{array}{l}0.624 \\
1.078\end{array}$ & 0.786 & 0.629 & 0.461 \\
\hline
\end{tabular}

Fuente: Elaboración propia 
A partir de estos resultados es posible afirmar que el instrumento tiene consistencia interna y las variables encontradas son las más citadas por la literatura (ISO 26000, 2010; Jiménez, 2002), por lo cual también es posible afirmar que tiene validez de constructo. Con estas variables se procedió a revisar su normalidad a través de la prueba de Kolmogorov - Smirnov que arrojo que los datos obedecían a una distribución no normal y se obtuvieron sus datos descriptivos.
La totalidad de los datos descriptivos resultaron por arriba del punto medio de la escala, a excepción de la variable de medio ambiente, situación digna de revisar ya que en teoría las instituciones de educación superior le han dedicado esfuerzos y recursos a esta variable. Se realizó corrió una prueba de correlación para conocer la asociación interna entre las variables.

Tabla 4.

Datos descriptivos

\begin{tabular}{|c|c|c|c|c|c|c|}
\hline Variables & Media & Mediana & Moda & Desv. Estand. & Varianza & Rango \\
\hline Transparencia & 3.5 & 3.7 & 4.13 & 0.9988 & 0.998 & 3.87 \\
\hline Derechos humanos & 3.4 & 3.7 & 4.14 & 1.0550 & 1.113 & 3.86 \\
\hline Prácticas laborales & 3.6 & 3.7 & 4.00 & 1.0092 & 1.018 & 4.00 \\
\hline Prácticas justas de operación & 3.5 & 3.7 & 4.00 & 1.0293 & 1.059 & 4.00 \\
\hline Medio ambiente & 3.3 & 3.5 & 2.75 & 1.1009 & 1.212 & 4.00 \\
\hline Práctica de recursos humanos & 3.4 & 3.5 & 3.50 & 1.0521 & 1.107 & 4.00 \\
\hline Participación activa y desarrollo de la comunidad & 3.6 & 3.7 & 3.67 & 1.0528 & 1.108 & 4.00 \\
\hline Vinculación & 3.5 & 3.7 & 4.00 & 1.0748 & 1.155 & 4.00 \\
\hline Extensión & 3.6 & 4.0 & 4.00 & 1.0196 & 1.040 & 4.00 \\
\hline Asunto de los usuarios & 3.4 & 3.5 & 4.00 & 1.1887 & 1.413 & 4.00 \\
\hline
\end{tabular}

Fuente: Elaboración propia

Para conocer la causalidad de las variables se realizó una regresión múltiple, y arrojo los siguientes valores:

Tabla 6.

Regresión lineal múltiple

\begin{tabular}{|c|c|c|c|c|c|c|c|c|c|c|}
\hline & TRA & $\mathrm{DH}$ & PL & PJO & MA & PRH & PADC & VIN & EXT & ASC \\
\hline $\begin{array}{l}\text { Durbin } \\
\text { Watson }\end{array}$ & 2.273 & 2.452 & 2.115 & 1.779 & 2.178 & 1.708 & 2.246 & 1.808 & 1.983 & 2.317 \\
\hline $\mathbf{R}$ & 0.748 & 0.483 & 0.487 & 0.539 & 0.580 & 0.352 & 0.434 & 0.508 & 0.749 & 0.626 \\
\hline$F$ & 11.409 & 2.732 & 2.805 & 3.680 & 4.571 & 1.272 & 2.093 & 3.136 & 11.529 & 5.804 \\
\hline Sig & 0.000 & 0.008 & 0.006 & 0.001 & 0.000 & 0.265 & 0.039 & 0.003 & 0.000 & 0.000 \\
\hline Constante & $\begin{array}{l}0.543 \\
0.300\end{array}$ & $\begin{array}{l}1.337 \\
0.065\end{array}$ & $\begin{array}{l}1.405 \\
0.041\end{array}$ & $\begin{array}{l}1.584 \\
0.019\end{array}$ & $\begin{array}{l}0.192 \\
0.786\end{array}$ & $\begin{array}{l}2.785 \\
0.000\end{array}$ & $\begin{array}{l}1.630 \\
0.020\end{array}$ & $\begin{array}{l}1.678 \\
0.028\end{array}$ & $\begin{array}{l}0.371 \\
0.487\end{array}$ & $\begin{array}{l}-0.294 \\
0.689\end{array}$ \\
\hline TRA & & $\begin{array}{l}-0.009 \\
0.952\end{array}$ & $\begin{array}{l}0.042 \\
0.778\end{array}$ & $\begin{array}{l}0.056 \\
0.702\end{array}$ & $\begin{array}{l}0.165 \\
0.271\end{array}$ & $\begin{array}{l}-0.228 \\
0.165\end{array}$ & $\begin{array}{l}0.028 \\
0.861\end{array}$ & $\begin{array}{l}0.059 \\
0.702\end{array}$ & $\begin{array}{l}0.603 \\
0.000\end{array}$ & $\begin{array}{l}0.321 \\
0.037\end{array}$ \\
\hline $\mathrm{DH}$ & $\begin{array}{l}-0.005 \\
0.952\end{array}$ & & $\begin{array}{l}0.180 \\
0.088\end{array}$ & $\begin{array}{l}-0.008 \\
0.939\end{array}$ & $\begin{array}{l}0.305 \\
0.004\end{array}$ & $\begin{array}{l}-0.045 \\
0.702\end{array}$ & $\begin{array}{l}0.111 \\
0.330\end{array}$ & $\begin{array}{l}-0.041 \\
0.712\end{array}$ & $\begin{array}{l}0.100 \\
0.216\end{array}$ & $\begin{array}{l}-0.117 \\
0.293\end{array}$ \\
\hline PL & $\begin{array}{l}0.024 \\
0.778 \\
\end{array}$ & $\begin{array}{l}0.198 \\
0.088\end{array}$ & & $\begin{array}{l}0.108 \\
0.229\end{array}$ & $\begin{array}{l}0.280 \\
0.012\end{array}$ & $\begin{array}{l}0.096 \\
0.439\end{array}$ & $\begin{array}{l}-0.042 \\
0.727\end{array}$ & $\begin{array}{l}0.321 \\
0.005\end{array}$ & $\begin{array}{l}0.009 \\
0.913\end{array}$ & $\begin{array}{l}-0.077 \\
0.511\end{array}$ \\
\hline PJO & $\begin{array}{l}0.033 \\
0.072\end{array}$ & $\begin{array}{l}-0.009 \\
0.939\end{array}$ & $\begin{array}{l}-0.137 \\
0.226\end{array}$ & & $\begin{array}{l}0.276 \\
0.015\end{array}$ & $\begin{array}{l}0.018 \\
0.884\end{array}$ & $\begin{array}{l}-0.101 \\
0.406\end{array}$ & $\begin{array}{l}0.381 \\
0.001\end{array}$ & $\begin{array}{l}-0.036 \\
0.677\end{array}$ & $\begin{array}{l}0.231 \\
0.049\end{array}$ \\
\hline MA & $\begin{array}{l}0.090 \\
0.271\end{array}$ & $\begin{array}{l}0.324 \\
0.004\end{array}$ & $\begin{array}{l}0.270 \\
0.012\end{array}$ & $\begin{array}{l}0.258 \\
0.015\end{array}$ & & $\begin{array}{l}-0.008 \\
0.946\end{array}$ & $\begin{array}{l}0.105 \\
0.374\end{array}$ & $\begin{array}{l}-0.135 \\
0.239\end{array}$ & $\begin{array}{l}-0.132 \\
0.112\end{array}$ & $\begin{array}{l}0.159 \\
0.165\end{array}$ \\
\hline PRH & $\begin{array}{l}-0.014 \\
0.165\end{array}$ & $\begin{array}{l}-0.040 \\
0.702\end{array}$ & $\begin{array}{l}0.077 \\
0.439\end{array}$ & $\begin{array}{l}0.014 \\
0.884\end{array}$ & $\begin{array}{l}-0.007 \\
0.946\end{array}$ & & $\begin{array}{l}0.192 \\
0.071\end{array}$ & $\begin{array}{l}-0.149 \\
0.152\end{array}$ & $\begin{array}{l}0.064 \\
0.399\end{array}$ & $\begin{array}{l}0.146 \\
0161\end{array}$ \\
\hline PADC & $\begin{array}{l}0.014 \\
0.861\end{array}$ & $\begin{array}{l}0.105 \\
0.330\end{array}$ & $\begin{array}{l}-0.036 \\
0.727\end{array}$ & $\begin{array}{l}-0.085 \\
0.406\end{array}$ & $\begin{array}{l}-0.126 \\
0.239\end{array}$ & $\begin{array}{l}0.207 \\
0.071\end{array}$ & & $\begin{array}{l}-0.033 \\
0.764\end{array}$ & $\begin{array}{l}0.128 \\
0.103\end{array}$ & $\begin{array}{l}0.061 \\
0.578\end{array}$ \\
\hline VIN & $\begin{array}{l}0.031 \\
0.702\end{array}$ & $\begin{array}{l}-0.041 \\
0.712\end{array}$ & $\begin{array}{l}0.291 \\
0.005\end{array}$ & $\begin{array}{l}0.334 \\
0.001\end{array}$ & $\begin{array}{l}-0126 \\
0.239\end{array}$ & $\begin{array}{l}-0169 \\
0.152\end{array}$ & $\begin{array}{l}-0.034 \\
0.764\end{array}$ & & $\begin{array}{l}0.040 \\
0.622\end{array}$ & $\begin{array}{l}0.032 \\
0.774\end{array}$ \\
\hline EXT & $\begin{array}{l}0.532 \\
0.000\end{array}$ & $\begin{array}{l}0.188 \\
0.216\end{array}$ & $\begin{array}{l}0.016 \\
0.913\end{array}$ & $\begin{array}{l}-0.060 \\
0.677\end{array}$ & $\begin{array}{l}-0.233 \\
0.112\end{array}$ & $\begin{array}{l}0.137 \\
0.399\end{array}$ & $\begin{array}{l}0.253 \\
0.103\end{array}$ & $\begin{array}{l}0.075 \\
0.622\end{array}$ & & $\begin{array}{l}0.288 \\
0.057\end{array}$ \\
\hline ASC & $\begin{array}{l}0.164 \\
0.037\end{array}$ & $\begin{array}{l}-0.116 \\
0.293\end{array}$ & $\begin{array}{l}-0.070 \\
0.511\end{array}$ & $\begin{array}{l}0.203 \\
0.049\end{array}$ & $\begin{array}{l}0.149 \\
0.165\end{array}$ & $\begin{array}{l}0.165 \\
0.161\end{array}$ & $\begin{array}{l}0.063 \\
0.578\end{array}$ & $\begin{array}{l}0.032 \\
0.774\end{array}$ & $\begin{array}{l}0.153 \\
0.057\end{array}$ & \\
\hline
\end{tabular}

Fuente: Elaboración propia 
A partir de estos resultados se puede establecer que las causalidades de las variables se explican a partir de las ecuaciones:

$\mathrm{TRA}=0.582(\mathrm{EXT})+0.164(\mathrm{ASC})$

$\mathrm{DH}=0.324(\mathrm{MA})$

$\mathrm{PL}=1.405+0.270(\mathrm{MA})+0.291(\mathrm{VIN})$

$\mathrm{PJO}=1.584+0.258(\mathrm{MA})+0.334(\mathrm{VIN})+0.203(\mathrm{ASC})$

$M A=0.305(D H)+0.280(P L)+0.276(P J O)$

$\mathrm{PRH}=2.785$

$\mathrm{PADC}=1.630$

$\mathrm{VIN}=1.678+0.321(\mathrm{PL})+0.381(\mathrm{PJO})$

EXT $=0.603($ TRA $)$

ASC $=0.321(T R A)+0.231($ PJO $)$
Es de llamar la atención que las variables de prácticas de recursos humanos y la participación activa y el desarrollo de la comunidad son iguales a una constante, lo que indica que esas dos variables no dependen del resto de variables planteadas.

Se realizaron comparativos por género con la intención de conocer si las variables encontradas muestran alguna diferencia en su percepción producto del género.

Tabla 7.

Comparativo por género

\begin{tabular}{|c|c|c|c|c|c|c|c|c|c|c|}
\hline & TRA & $\mathrm{DH}$ & PL & PJO & MA & PRH & PADC & VIN & EXT & ASC \\
\hline $\begin{array}{l}\text { Rangos } \\
\text { Mas } \\
\text { Fem }\end{array}$ & $\begin{array}{l}50.17 \\
41.73\end{array}$ & $\begin{array}{l}53.78 \\
38.09\end{array}$ & $\begin{array}{l}49.12 \\
42.08\end{array}$ & $\begin{array}{l}51.45 \\
40.43\end{array}$ & $\begin{array}{l}50.43 \\
41.47\end{array}$ & $\begin{array}{l}40.48 \\
51.64\end{array}$ & $\begin{array}{l}45.88 \\
46.12\end{array}$ & $\begin{array}{l}51.03 \\
40.86\end{array}$ & $\begin{array}{l}43.53 \\
48.52\end{array}$ & $\begin{array}{l}46.38 \\
45.61\end{array}$ \\
\hline $\begin{array}{l}\text { Suma } \\
\text { Mas } \\
\text { Fem }\end{array}$ & $\begin{array}{l}2308 \\
2878\end{array}$ & $\begin{array}{l}2474 \\
1712\end{array}$ & $\begin{array}{l}2259.5 \\
1926.5\end{array}$ & $\begin{array}{l}2366.5 \\
1819.5\end{array}$ & $\begin{array}{l}2320 \\
1866\end{array}$ & $\begin{array}{l}1862 \\
2324\end{array}$ & $\begin{array}{l}2110.5 \\
1075.5\end{array}$ & $\begin{array}{l}2347.5 \\
1838.5\end{array}$ & $\begin{array}{l}2002.5 \\
2183.5\end{array}$ & $\begin{array}{l}2133.5 \\
2052.5\end{array}$ \\
\hline $\begin{array}{l}\text { U Mann } \\
\text { Whitney }\end{array}$ & 843 & 677 & 891.4 & 784.5 & 831 & 781 & 1029.5 & 803.5 & 921.5 & 1017.5 \\
\hline W Wilcox & 2878 & 1712 & 1926.5 & 1819.5 & 1866 & 1862 & 2110.5 & 1838.5 & 2002.5 & 2052.5 \\
\hline Z & -1.527 & -2.847 & -1.143 & -1.996 & -1.629 & -2.023 & 0.044 & 1.849 & -0.907 & -0.140 \\
\hline sig & 0.127 & 0.004 & 0.253 & 0.046 & 0.104 & 0.043 & 0.956 & 0.064 & 0.365 & 0.889 \\
\hline
\end{tabular}

Fuente: Elaboración propia

El comparativo $U$ de Mann Whitney establece que existen diferencias en la percepción de las variables de derechos humanos, prácticas de derechos humanos y vinculación.

De igual forma se ejecutó una prueba para establecer las posibles diferencias de percepción producto del grado académico

Tabla 8.

Comparativo por grado académico

\begin{tabular}{|c|c|c|c|c|c|c|c|c|c|c|}
\hline & TRA & DH & $\mathrm{PL}$ & PJO & MA & PRH & PADC & VIN & EXT & ASC \\
\hline \multicolumn{11}{|l|}{ Rangos } \\
\hline Lic & 46.10 & 46.94 & 43.41 & 50.95 & 45.80 & 46.19 & 47.81 & 49.53 & 47.48 & 48.01 \\
\hline Ms & 50.11 & 41.48 & 50.50 & 44.63 & 43.67 & 49.35 & 37.39 & 46.00 & 47.52 & 49.93 \\
\hline $\mathrm{Dr}$ & 38.86 & 49.82 & 48.61 & 29.93 & 39.79 & 39.79 & 53.14 & 32.39 & 37.79 & 31.79 \\
\hline $\begin{array}{l}\text { Chi - } \\
\text { Cuadrado }\end{array}$ & 1587 & 1038 & 1334 & 7042 & 1155 & 3770 & 4735 & 4735 & 1620 & 4964 \\
\hline gl & 2 & 2 & 2 & 2 & 2 & 2 & 2 & 2 & 2 & 2 \\
\hline Sig asint & 0.452 & 0.595 & 0.513 & 0.030 & 0.737 & 0.561 & 0.152 & 0.094 & 0.445 & 0.084 \\
\hline
\end{tabular}

Fuente: Elaboración propia

El comparativo $\mathrm{H}$ de Kruskal Wallis mostro diferencias por concepto de grados académicos, en las variables de prácticas justas de operación, vinculación y asunto de los consumidores.

Finalmente se analizó la diferencia de las variables por concepto de tipo de institución de educación. 
A través de esta prueba es evidente que entre instituciones privadas y públicas la diferencia de percepción se hizo evidente solamente en la variable de prácticas justas de operación.

Tabla 9. Comparativo por tipo de institución

\begin{tabular}{l|l|l|l|l|l|l|l|l|l|l}
\hline & TRA & DH & PL & PJO & MA & PRH & PADC & VIN & EXT & ASC \\
\hline $\begin{array}{l}\text { Rangos } \\
\text { Publica }\end{array}$ & 45.65 & 45.65 & 44.62 & 42.28 & 43.85 & 46.82 & 44.87 & 45.66 & 47.75 & 46.33 \\
Privada & 46.88 & 47.10 & 49.44 & 54.81 & 51.37 & 43.94 & 48.87 & 46.85 & 41.63 & 45.17 \\
\hline $\begin{array}{l}\text { Suma } \\
\text { Publica }\end{array}$ & 2967 & 2961.5 & 2900.5 & 2761 & 2850.5 & 3043.5 & 2916.5 & 2968 & 3103.5 & 3011.5 \\
Privada & 1219 & 1224.5 & 1285.5 & 1425 & 1335.5 & 1142.5 & 1269.5 & 1218 & 1082.5 & 1174.5 \\
\hline $\begin{array}{l}\text { U Mann } \\
\text { Whitney }\end{array}$ & 822 & 816.5 & 755.5 & 616 & 705.5 & 791.5 & 771.5 & 823 & 731.5 & 823.5 \\
\hline W Wilcox & 2967 & 2961.5 & 2900.5 & 2761 & 2850.5 & 1142.5 & 2916.5 & 2968 & 1082.5 & 1174.5 \\
\hline Z & -0.202 & -0.251 & -0.789 & -2.019 & -1.229 & -0.472 & -0.650 & -0.194 & -0.1003 & -0.191 \\
\hline sig & 0.840 & 0.802 & 0.430 & $\mathbf{0 . 0 4 3}$ & 0.219 & 0.637 & 0.517 & 0.846 & 0.316 & 0.849 \\
\hline Fun
\end{tabular}

Fuente: Elaboración propia

\section{CONCLUSIONES}

A partir de esta investigación, fue posible diseñar un instrumento que obedece a las variables de responsabilidad social en instituciones de educación superior, que se señalan en la literatura, como lo son las dimensiones de la ISO 26000 (2010), se evidenciaron también dos dimensiones de la propuesta original de Jimenez (2002) popularizada por Vallaeyes, De la Cruz y Saisa (2006), la vinculación y la extensión; si tomamos en cuenta que la responsabilidad social comienza cuando las obligaciones propia de la actividad ya ha sido solventada, las otras dos dimensiones de esta teoría, docencia e investigación, propiamente no serían elementos de responsabilidad social, ya que las instituciones de educación superior tienen la obligación de fomentarlas y verificar que se realicen.

Otra variable, a que emergió fue el de las prácticas de recursos humanos, ya que estudios han evidenciado, dentro de las instituciones de educación superior, prácticas de precarización del trabajo (Álvarez, Ojeda y Sánchez, 2017; Álvarez, Ojeda y López, 2018) y esta práctica es uno de los grandes pendientes de las instituciones de educación superior en cuanto a responsabilidad social.

Las variables propuestas demostraron consistencia y validez interna, dado que los índices superaron los establecidos por la literatura (Hair, Anderson, Tatham y Black, 1999), de igual forma las correlaciones demostraron ausencia de problemas de colinealidad (Kleinbaum, Kupper y Muller, 1988; Belsley, 1991).
En cuanto a las regresiones se obtuvo un sistema de ecuaciones a través de los cuales, cada una de las variables se puede explicar en función a las otras, a excepción de las prácticas de recursos humanos y la participación activa y el desarrollo de la comunidad, lo que nos indicaría, que, en la percepción de la muestra encuestada, estas dos variables son independientes del resto de las variables de responsabilidad social propuesta.

La prueba de $U$ de Mann Whitney, en lo que respecta a género, evidencio diferencias significativas en las variables de derechos humanos, prácticas laborales y vinculación, lo que puede significar que los participantes de la muestra perciben que continúan existiendo prácticas que fomentan la diferenciación, exclusión o discriminación en estas tres variables.

La misma prueba de contrastes de medianas, en cuanto al tipo de institución, pública o privada, solo evidencio diferencias en la variable de prácticas justas de operación, situación que puede ser explicada por la naturaleza de la institución, las públicas tienen que responder a lógicas de gobierno y las privadas a la lógica del mercado de la iniciativa privada, por lo que no fue sorpresa encontrar que en esta variable se visualizan esas diferencias.

Finalmente, en el contraste realizado por grado académico, las diferencias fueron encontradas en las variables de prácticas justas de operación, vinculación y asunto de los consumidores, situación que es posible explicar debido a la naturaleza de los alumnos a los que cada uno de estos niveles imparten clases, no es igual las necesidades y 
percepciones de las necesidades de alumnos de pregrado que de alumnos de posgrado, como tampoco lo es igual entre alumnos de maestría y de doctorado.

Se considera que el presente estudio aporta a la responsabilidad social en instituciones de educación superior, toda vez que ofrece un instrumento cuantitativo con el cual se podría medir la responsabilidad social en esas organizaciones (ver anexo), desde un concepto holístico del mismo constructo, integrando las teorías que han emergido al respecto desde Jiménez (2002), Larran y Andrades (2015), De la Cruz y Saisa (2008), Rodriguez (2010), ISO 26000 (2010) y Gaete (2011), sin embargo, al ser un primer ejercicio los resultados deben considerarse desde la particularidad del presente estudio y verificarse en otros contextos, con otras poblaciones y otras muestras.

\section{REFERENCIAS:}

Aldeanueva, I. y Arrabal, G. (2018). A comunicacao e mensuracao da responsabilidade social universitaria: redes sociais e proposta de indicadores. Revista digital de investigación en docencia universitaria. 12 (1): 121 -136.

Álvarez, D., Ojeda, J. y López, C. (2018). Factors that determine work precariousness - the compariosn between the national and foreing direct investment industries: the case of Celaya, Guanajuato, México. International Journal of Management Excellence. 11(2): 1563 - 1573

Álvarez, D., Ojeda, J. y Sánchez, M. (2017). 0 acceso as presetacions laboráis minimas en Celaya (Guanajuato, México) baixo unha perspectiva de xénero. Anuario da Facultade de Ciencias do Traballo da Universidade da Coruña. 8:13-32

Arocena, R. y Sutz, J. (2001). Mirando los sistemas nacionales de innovación desde el sur. Sala de lectura CTS+I. Organización de estados lberoamericanos. http://www.oei.es/salactsi/sutzarcena.htm

Arredondo, M. y Ojeda, J. (2015). Responsabilidad social universitaria en el estado de Guanajuato. En Posada, R., Aguilar, O. y Ojeda, J. (Coord). Propuestas universitarias para la responsabilidad social. 39 - 70. Guanajuato, México. Ediciones de la Universidad Politécnica de Guanajuato.

Atakan, S. y Eker, T. (2007). Corporate identity of a socially responsable university: a case from the Turkish higher education sector. Journal of Business Ethics. 76(1):55-68.

Belsley, D. (1991). Conditioning diagnostics: Collinearity and weak data in regression. John Wiley and Sons Ed.

Benneworth, P. (2013). University Engagement with socially excluded communities Netherlands: Springer
Boelen, C. Responsabilidad social y excelencia. Educación médica. 12(4):199 - 205

Buchan, G., Spellerberg, I. y Blum, W. (2007). Education for sustainability. Developing a posgraduate level subjet whit and international persperctive. International Journal of Sustainbility in Higher Education. 8(1):4 - 15

Campbell, M. y Christie, M. (2008). Indigenous community engagement. Casuarina Australia: Charles Darwin University. Office of the pro vice chancellor community and Access.

Cano, C. A. G., Castillo, V. S., Fajardo, M. Y., Trucco, G. G. G., \& Garzón, D. C. (2017). Caracterización de los graduados del programa de contaduría pública de la universidad de la amazonia 20132015. FACE: Revista de la Facultad de Ciencias Económicas y Empresariales, 16(2), 29-37.

Castañeda, G., Ruíz, M., Viloria, O., Castañeda, R. y Quevedo, Y. (2007). El rol de las universidades en el contexto de la responsabilidad social empresarial. Revista Negotium, 3(8): 100 - 132.

Castillo, I. (2010). Una aproximación al estado de la RSE en la enseñanza de España. Madrid: Club de la Excelencia en Sostenibilidad, Ministerio del Trabajo e Inmigración. CEMEX, España.

Cavero, L. (2006). La responsabilidad social universitaria: Transformaciones para el Perú y América Latina. Pontificia Universidad Católica del Perú. http://blog.pucp.edu.pe/index.php

Centeno, P. (2006). El rol de las universidades en contexto de la responsabilidad social empresarial. Revista Negotium, http://www.revistanegotium.org.ve/pdf/8/Art4.pdf.

Ciurana, G. y Filho, W. (2006). Education for sustainablitiy in university studies. Experiences from Project involving European and latin American universities. International Journal of Sustainability in Higher Education. 7(1): 81 - 93

Cruz, J., Rositas, J. y García, J. (2013). Responsabilidad social empresarial: investigación empírica exploratoria sobre los conceptos de RSE y RSU. XVIII Congreso Internacional de Contaduría, Administración e Informática, México, D.F. 2, 3 y 4 de Octubre 2013.

Davidovich, M., Espina, A., Navarro, G. y Salazar, L. (2005). Construcción y estudio piloto de un cuestionario para evaluar comportamientos socialmente responsables en estudiantes universitarios. Revista de Psicología. 14(1): 125 139.

De la Cruz, C. y Saisa, P. (2008). La responsabilidad de la universidad en el proyecto de construcción de una sociedad. Revista Educación Superior y Sociedad. 13(2):17-52 
Delgado, R. (2007). Redimensionando el concepto de responsabilidad social. http://www.anahuac.mx/clares/articulos.018.html

Dias, J. (2008). Calidad, pertinencia y relevancia: relación con el resto del sistema y la sociedad, responsabilidad social de la educación superior. En Gazzola, A. y Pires, S. (Coord). Hacia una política regional de aseguramiento de la calidad en la educación superior para América Latina y el Caribe. Caracas: UNESCO - IESALC.

Díaz, M. y Facal, S. (2011). Percepciones de los estudiantes de la facultad de ciencias empresariales sobre la responsabilidad social universitaria (Montevideo - Uruguay). Investigación y Desarrollo. 19(2):340-365.

Domínguez, M. y López, E. (2009). Estudiantes universitarios opinan sobre la responsabilidad social universitaria. Humanismo y Trabajo Social. $8: 223-246$

Dupas, G. (2005). Atores e poderes na nova orden global. Assimetrias, instabilidades e imperativos de legitimacao. Sao Paulo. Editoria UNESP

Ernült, J. (2011). Responsabilidad social en la universidad. IX Congreso Internacional de Análisis Organizacional. Conferencia Magistral. San Luis Potosí, SLP.

Ferrer-Balas, D., Adachi, J., Banas, S., Davidson, C., Hosikoshi, A., Mishra, A., Motoda, Y, Onga, M. y Ostwald, M. (2008). An international comparative analysis of sustainability transformation across seven universities. International Journal of Sustainability in Higher Education. 9(3): 295 316.

Gaete, R. (2011). Responsabilidad social universitaria: una nueva mirada a la relación de la universidad con la sociedad desde la perspectiva de las partes interesadas. Un estudio de caso. PhD thesis. Departamento de Sociología y Trabajo Social. Universidad de Valladolid.

García, C. y Alvarado, Y. (2012). Responsabilidad social universitaria como plataforma para el desarrollo de la comunidad. Multiciencias. Vol. 12 enero diciembre. $59-63$

Gargantini, D. (2019). Directorio de homólogos participantes. AUJAL. https://www.ausjal.org/responsabilidad-socialuniversitaria

Gómez, O., De Pelekais, C., y Tirado, L. (2009). Responsabilidad social y formación gerencial del personal docente en las universidades nacionales. Omnia. 15(3): 198 - 208.

Gordon, I. y Geraldi, M. (2005). Factors thata affect undertanding of social responsibility accounting. Canadian Accounting Perspectives. 4(1): 31 - 59.
Guédez, V. (2003). Seminario internacional. Universidad Metropolitana. Gobernabilidad social, ética y políticas públicas. Caracas, Venezuela, OPSU.

Guédez, V. (2006). Ética y práctica de la responsabilidad social empresarial. Editorial Planeta. Caracas Venezuela.

Hair, J., Anderson, R., Tatham, R. y Black, W. (1999). Análisis multivariante. Ed Pearson, México

Hammond, C., y Churchman, D. (2008). Sustaining academic life: A case for applying principles of social sustainability to the academic profession. International Journal of Sustainability in Higher Education. 9(3): 235 - 245.

Hammurabi (1750 a.C.). Código de Hammurabi. http://www.ataun.net/BIBLIOTECAGRATUITA/Cl \%C3\%A1sicos $\% 20$ en $\% 20$ Espa $\%$ C3\%B1ol/An\%C 3\%B3nimo/C\%C3\%B3digo\%20de\%20Hammurab i.pdf

Hernández, R. y Saldarriaga, A. (2009). Gestión de la responsabilidad social universitaria: caso de la escuela de ingeniería de Antioquia. Dyna. 76(159): $237-248$

Hill, R. (2004). The social - responsible university: talking the talk while walking the walk in the college of business. Journal of Academic Ethics. 2: 89 - 100

Holdsworth, S., Wyborn, C. y Bekessy, S. y Thomas, I. (2008). Professional development for education for sustainability, How advanced are australian universties?. International Journal of Sustainability in Higher Education. 9(2):131 146.

Ibrahim, N., Angelidis, J. y Howard, D. (2006). Corporate social responsibility: A comparative analysis of perceptions of practicing accountants and accounting students. Journal of Business Ethics. 66: $157-167$.

ISO 26000 (2010). ISO 26000 social responsibility. http://www.iso.org/iso/sr 7 core subjects.pdf

Jiménez, M. (2002). La Universidad Construye País. Corporación participa. Ponencia presentada en Seminario realizado en Valparaiso, Chile, http://rsuniversitaria.org/web/images/stories/mem oria/UCP\%202002.pdf

Jiménez, M., De Ferari, J., Del Piano, C. y Andrade, L. (2004). Universidad construye país. Documento de trabajo. www.construyepais.cl

Kleinbaum, D., Kupper, L. y Müller, K. (1988). Applied regression analysis and other multivariable methods. PWS Publishing Co. Boston, MA, USA.

Larran, M. y Andrades, F. (2015). Análisis de la responsabilidad social universitaria desde diferentes enfoques teóricos. Revista Iberoaméricana de Educación Superior. 6(15):91 $-107$

Martínez, C., Mavarez, L., Rojas, J., Rodríguez, y Carvallo, B. (2008). La responsabilidad social como 
instrumento para fortalecer la vinculación universidad - entorno social. Trabajo presentado en el I Congreso Iberoamericano de Ciencia, Tecnología, Sociedad e Innovación, CTS + I. Academia Mexicana de Ciencias y la Academia de Ingenieros.

Matten, D. y Moon, J. (2004). Corporate social responsibility education in Europe. Journal of Business Ethics. 54: 323 - 337.

Montaño, L. (2012). Organización, discurso y compromiso, reflexiones acerca de los programas de responsabilidad social. En Morales, E., Navarro, G. y Santa Ana, M. (Coord). Sustentabilidad, responsabilidad social y organizaciones, dimensiones, retos y discursos. Editorial Hess.

Montgomery, D. y Ramus, C. (2003). Corporate social responsaibility reputation effects on MBA job choice. Research Paper No. 1805. Stanford Graduate School of Business.

Ocegueda, J., Mungaray, A., Rubio, J. y Moctezuma, P. (2016). La responsabilidad social de la universidad mexicana a mitad del siglo XXI. Editorial Porrúa. Universidad Autónoma de Baja California.

Ojeda, J. y Álvarez, D. (2015). Responsabilidad social en las universidades: antecedentes, trayectorias y perspectivas. Revista COEPES. Año 4, No. 12.

Ojeda, J. (2013a). Responsabilidad social, construcción de un concepto desde la percepción del docente universitario. Revista científica teorías, enfoques y aplicaciones en las ciencias sociales. 5(12): 11 $-24$.

Ojeda, J. (2013b). Responsabilidad social del docente universitario. Un acercamiento desde los derechos humanos. En González, T. y Martín, A. (Coords). Análisis de la responsabilidad social en el siglo XXI. México: Universidad De La Salle.

Ojeda, J. (2014). Responsabilidad social. La práctica desde la universidad. En Mancilla, E. y Ojeda, J. (Coord). Desarrollo sustentable, logística y desarrollo de nuevos proyectos, aproximaciones, abordajes y experiencias. 107 - 124. México: Universidad De La Salle.

Ojeda, J. (2015a). Responsabilidad social universitaria, una visión multidisciplinaria. Conferencia Universidad Iberoamericana - Leon, Noviembre.

Ojeda, J. (2015b). La responsabilidad social en empresas trasnacionales. Conferencia Universidad Autónoma Metropolitana - Azcapotzalco, Julio.

Ojeda, J. (2018). La responsabilidad social: Los contratos de trabajo y los stakeholders, un acercamiento desde los trabajos de Begnt Hölstrom. Gestión y estrategia, 53(29: $55-68$.

Ojeda, J. y Álvarez, D. (2015). Responsabilidad social en las universidades: antecedentes, trayectorias y perspectivas. Revista COEPES, Guanajuato. Mayo.

Ojeda, J. y Lira, G. (2014). Responsabilidad social. En Conraud, E. (Coord). Responsabilidad social en empresas multinacionales en el estado de Guanajuato. Ed. Pearson.

Ojeda, J., Álvarez, D. y Arredondo, M. (2018). La relación de los valores en la generación de responsabilidad social, en una institución privada de educación superior. En Muñoz, B. (Coord). La diversidad y el desarrollo social desde la comunicación. 67 - 90. Cuadernos artesanos de comunicación 152. Santiago de Compostela, España.

Ojeda, J., Silva, M. y Álvarez, D. (2015). La responsabilidad social en la universidad. Una mirada desde el alumnado. Hunab Ku / Economía y Negocios. $5: 81-104$

Ojeda, J., Vega, M. y Vera, J. (2017). Tendencias de la responsabilidad social en instituciones de educación superior: confrontando paradigmas. En De la Rosa, M. (Coord) Prácticas y tendencias del desarrollo sustentable y la responsabilidad social, pp. $223-281$.

Pérez, J. y Vallaeys, F. (2016). Prácticas y modelos de responsabilidad social universitaria en México. Proceso de transformación en las universidades.ANUIES. Universidad Autónoma de Yucatán.

Rendueles, M. (2010). Mercado social, responsabilidad social y balance social. Conceptos a desarrollar por instituciones universitarias. Telos, Revista de estudios interdisciplinarios de ciencias sociales. 12(1):29- 42

Rodríguez, J. (2010). Responsabilidad social universitaria: del discurso simbólico a los desafíos reales, pp. 3 - 24. Netbiblio. La Coruña, España

Sánchez, C., Herrera, A., Zarate, L. y Moreno, W. (2007). La responsabilidad social universitaria (RSU) en el contexto del cambio de la educación superior. Virtual Educa Brasil. https://reposital.cuaed.unam.mx:8443/xmlui/bitstr eam/handle/123456789/1457/225MSG.PDF?sequence $=1$

Sanje, G. y Senol, I. (2012). Corporate social responsibility in higher education institutions: Istanbul Bilgi University Case. American International Journal of Contemporary Research. 2(3): $95-103$

Setó - Paimes, D., Domingo - Vernis, M. y Rabassa Figueras, N. (2011). Corporate social responsibility in management education: current status in spanish universities. Jorunal of Management and Organization. 17(5): $604-620$.

Tünnermann, C. (2003). El nuevo concepto de la extensión universitaria, en la Universidad Latinoamericana ante los retos del siglo XXI, México. 
Ur - Nammu (2100 a.C.). Código de Ur Nammu. http://www.comuna.cat/Glosario/Codigo de_UrNammu.pdf

Urzúa, R. (2001). La responsabilidad social de las universidades: una guía de discusión. Documento presentado en el taller, elaboración de estrategia para la expansión de la responsabilidad social en las universidades chilenas. Santiago, 4 y 5 de octubre 2001.

Vallaeyes, F. (2008). Responsabilidad social universitaria: Una nueva filosofía de gestión ética e inteligente para las universidades. Educación superior y sociedad. 12(2):193-219. http://unesdoc.unesco.org/images/0018/001820/1 82067s.pdf

Vallaeys, F. (2005). La responsabilidad social de la universidad. En en: http://palestra.pucp.edu.pe/index.php?id=111

Vallaeys, F. (2006). La responsabilidad social universitaria. Pontificia Universidad Católica del Perú. http://www.construyepais.cl

Vallaeys, F. (2007). Breve marco teórico de responsabilidad social universitaria. Universidad de las Américas de Puebla, Banco Interamericano de Desarrollo. http://www.udlap.mx/rsu/pdf/1/ BreveMarcoTeodelaResponsabilidad SocialUniv.pdf

Vallaeys, F. y Carrizo, L. (2006). Responsabilidad social universitaria: marco conceptual, antecedentes, herramientas. CD multimedia. Lima, Perú. Banco Interamericano de Desarrollo

Vallaeys, F., De la Cruz, C. y Saisa, P. (2009). Responsabilidad social universitaria. Manual primeros pasos. México D.F.: Mc Graw Hill

Zaratustra (1776 a.C.). Los Gathas. https://gathagatha.files.wordpress.com/2013/08/g athas -los-cantos-divinos-de-zaratustra-spanishedition.pdf 